\section{Molecular Syndromology}

Mol Syndromol 2017;8:279-281

DOI: $10.1159 / 000481332$
Accepted: August 30, 2017

by M. Schmid

Published online: October 11, 2017

\title{
Neocentromeres to the Rescue of Acentric Chromosome Fragments
}

Single or double breaks in the same chromosome arm will produce terminal or interstitial acentric fragments, respectively. These fragments will be lost during mitotic or meiotic division because they do not contain a centromere. Depending on their size, and consequently the number of genes affected, a lost acentric fragment may affect the phenotype or even not be compatible with viability of the carrier organism. If a new centromere, a neocentromere, is formed on an acentric fragment, it will segregate as a small supernumerary marker chromosome (sSMC) after mitotic or meiotic divisions. As of 2008, up to 90 cases of constitutional sSMCs containing neocentromeres have been documented [Marshall et al., 2008]. These consisted of either an inverted duplication (inv dup) of the distal part of a chromosome arm resulting in an unbalanced karyotype (class I) or a balanced chromosomal rearrangement after an interstitial deletion producing linear and circular sSMCs (class II). Class I sSMCs with a neocenromere make the carrier tetrasomic for the duplicated region or trisomic if the region distal to the duplicated region is deleted from the original chromosome. In cases of class II sSMCs with 2 breaks in either the same chromosome arm or opposite arms, the outcome is always balanced. With 2 breaks in the same chromosome arm, a supernumerary ring with the paracentric chromosome segment results, while breaks in opposite chromosome arms produce a pericentric ring chromosome. The class I inv dup sSMCs with neocentromeres comprise $74 \%$ (67 out of 90) of the reported cases [Marshall et al., 2008]. Chromosomes 3, 8, 13, and 15 are hotspots for neocentromeres containing class I sSMCs

\section{KARGER}

(C) 2017 S. Karger AG, Basel with breakpoints in regions 3q26q27.3, 8p22p23, 13q31q33.1, and 15q22q26, respectively [Marshall et al., 2008]. These breakpoint regions contain large blocks of segmental duplications which make them particularly prone to rearrangements, be it tandem duplications, deletions, or neocentromere containing sSMCs [Bailey and Eichler, 2006; Sharp et al., 2006]. This does not exclude other repetitive elements such as long interspersed nuclear elements and short interspersed nuclear elements, LINEs and SINEs, respectively, which have been found in neocentromeres containing sSMCs involving breaks in 3q11.2, 3q26.2, 5q14.3, 8p23.1, 8q23.3, 9p23, 9p13.1, 11q21, Xq12, and Xq22.1 [Murmann et al., 2009].

Individuals trisomic for the duplicated region of $8 \mathrm{p} 23$ exhibit a recognizable syndrome, regardless of whether a neocentromere containing class I sSMC has been formed [Barber et al., 2008, 2015; Murmann et al., 2009]. Ascertainment of sSMCs requires classical karyotyping followed by detailed molecular cytogenetic analyses, such as FISH and array CGH [Murmann et al., 2009; Burnside et al., 2011; Hochstenbach et al., 2013, 2016, 2017]. Thus, sSMCs were found in $0.043 \%$ of live births [Liehr et al., 2004]. A study of 241 unpublished sSMCs from 2005 suggested that neocentromeres are present in around $3 \%$ of the published cases of sSMCs [Dalprà et al., 2005]. A combined estimate of neocentromere formation on inverted duplicated chromosomes would be approximately once in every 70,000-200,000 live births. This number may be an overestimate since cases of sSMCs containing neocentromeres may be considered novel and highly interesting, thus leading to a positive publication bias [Marshall et al.,

Martin Poot

Department of Human Genetics, University of Würzburg Biozentrum, Am Hubland DE-97074 Würzburg (Germany)

E-Mail Martin_Poot@hotmail.com 
2008]. Therefore, a precise estimate of the frequency of germline neocentromere formation in the general population may be difficult to obtain.

Little is known as to how neocentromeres are being formed. No experimental formation of neocentromeres in cultured human cell lines has been reported, such that our current understanding is limited to medical genetic case reports, studies of tumors and model organisms as well as to observations of karyotype evolution in primates [Marshall et al., 2008; Burrack and Berman, 2012; Rocchi et al., 2012]. In a case with a neocentromere at band 3 q26.1 in a chromosome where the centromeric region was deleted, no alpha-satellite DNA was detected at the neocentromere, although it stained positive with anticentromere antibodies [Wandall et al., 1998]. However, clustering of neocentromeres at particular cytogenetic locations may not be as precise as the older literature has suggested [Alonso et al., 2003; Marshall et al., 2008; Hasson et al., 2011]. Thus far, no clear sequence specificity for neocentromere-containing regions has been reported, although a threshold AT-base pair content requirement cannot be discounted [Alonso et al., 2003; Marshall et al., 2008]. Interestingly, neocentromere-containing regions are among the earliest to be replicated during the cell cycle [Koren et al., 2010]. Once a new centromere has been formed, its position and boundaries need to be maintained by acquisition of satellite DNA, among other items. Since observations of clinical cases do not indicate that neocentromeres are located in specific DNA sequences, epigenetic marks, such as methylation of CpG islands, are a probable mechanism to achieve this [Marshall et al., 2008; Burrack and Berman, 2012]. While CpG islands longer that 200 nucleotides close to the neocentromere were generally hypermethylated in comparison to those in the same region in a chromosome without a neocentromere, the boundaries of the areas covered by the centromeric proteins were clearly hypomethylated [Wong et al., 2006]. In a study of 14 evolutionary new centromeres in macaque chromosome 4 , which is equivalent to human chromosome 6 , preferential neocentromere formation in gene deserts has been found [Ventura et al., 2007; Lomiento et al., 2008]. Thus, studies of evolutionary new chromosomes (ENC) may shed some light on the mechanism(s) of formation and subsequent fixation of centromeres.
Early comparative cytogenetic studies described a polymorphic orangutan chromosome 12 (chromosome 9 in the classical nomenclature) with a high allele frequency (over 20\%) in both Borneo (Pongo pygmaeus) and Sumatra (Pongo abelii) orangutan populations [Turleau et al., 1975; Seuánez et al., 1976; de Boer and Seuánez, 1982]. The marker order of the variant chromosome was identical to the normal homolog such that the polymorphism was probably due to a centromere repositioning event [Locke et al., 2011]. This ENC is unique since both the original centromere and the neocentromere coexist in the same population as a polymorphism, and this ENC was relatively young since it did not contain satellite DNA. Tolomeo et al. [2017] sequenced the genome of an orangutan homozygous for the ENC and did not find any significant variations relative to the wild-type counterpart. By measuring the interprobe distances between BACs $\mathrm{CH} 276-136 \mathrm{P} 13$ and $\mathrm{CH} 276-12 \mathrm{M} 5$, the ENC domain was found to be significantly more compact than the corresponding wild-type region. This indicates that compaction is an intrinsic property of centromeres, regardless of the presence of heterochromatin. If this stronger compaction affects gene expression, it may inhibit spreading of ENCs. This would depend on the importance of these gene(s) for viability of the carrier organism. Interestingly, the expression of the only gene embedded in the ENC region, SLC6A15 (Pongo abelii solute carrier family 6, neutral amino acid transporter, member 15; chr12: $85,450,585-85,510,678)$, appears repressed, probably as a consequence of the compaction of this locus. The SLC6A 15 gene appears to be disposable and thus does not act as a bias against the ENC spreading in the Pongo population. Incomplete repression of the SLC6A15 gene would suggest that it may not be completely superfluous. Conceivably, the activity of this gene has helped maintain this area in its original satellite-free condition after ENC seeding. The authors conclude that this finding is the first evidence that ENC seedings are epigenetic in nature. This study generated novel and unexpected insights into the possible mechanism(s) of origin of neocentromeres and opened up a new perspective on this still elusive process. Numerous questions remain to be addressed for which the present approach and techniques may provide valuable new avenues.

Martin Poot 


\section{References}

Alonso A, Hasson D, Cheung F, Warburton PE: A paucity of heterochromatin at functional human neocentromeres. Epigenetics Chromatin 3:6 (2010).

Bailey JA, Eichler EE: Primate segmental duplications: crucibles of evolution, diversity and disease. Nat Rev Genet 7:552-564 (2006).

Barber JC, Maloney VK, Huang S, Bunyan DJ, Cresswell L, et al: 8p23.1 duplication syndrome; a novel genomic condition with unexpected complexity revealed by array CGH. Eur J Hum Genet 16:18-27 (2008).

Barber JC, Rosenfeld JA, Graham JM, Kramer N, Lachlan KL, et al: Inside the 8p23.1 duplication syndrome; eight microduplications of likely or uncertain clinical significance. Am J Med Genet A 167A:2052-2064 (2015).

Burnside RD, Ibrahim J, Flora C, Schwartz S, Tepperberg $\mathrm{JH}$, et al: Interstitial deletion of proximal 8q including part of the centromere from unbalanced segregation of a paternal deletion/marker karyotype with neocentromere formation at $8 \mathrm{p} 22$. Cytogenet Genome Res 132:227-232 (2011).

Burrack LS, Berman J: Neocentromeres and epigenetically inherited features of centromeres. Chromosome Res 20:607-619 (2012).

Dalprà L, Giardino D, Finelli P, Corti C, Valtorta $\mathrm{C}$, et al: Cytogenetic and molecular evaluation of 241 small supernumerary marker chromosomes: cooperative study of 19 Italian laboratories. Genet Med 7:620-625 (2005).

de Boer LEM, Seuánez HN: The chromosomes of the orangutan and their relevance to the conservation of the species, in de Boer LEM (ed): The Orangutan: Its Biology and Conservation, vol 1, pp 135-170. (Dr. Junk Publishers, London 1982).

Hasson D, Alonso A, Cheung F, Tepperberg JH, Papenhausen PR, et al: Formation of novel CENP-A domains on tandem repetitive DNA and across chromosome breakpoints on human chromosome 8q21 neocentromeres. Chromosoma 120:621-632 (2011).
Hochstenbach R, van Gijn ME, Krijtenburg PJ, Raemakers R, van 't Slot R, et al: Gain of FAM $123 B$ and ARHGEF9 in an obese man with intellectual disability, congenital heart defects and multiple supernumerary ring chromosomes. Mol Syndromol 3:274-283 (2013).

Hochstenbach R, Nowakowska B, Volleth M, Ummels A, Kutkowska-Kaźmierczak A, et al: Multiple small supernumerary marker chromosomes resulting from maternal meiosis I or II errors. Mol Syndromol 6:210-221 (2016).

Hochstenbach R, Poot M, Liehr T: Mechanisms of origin and clinical effects of multiple small supernumerary marker chromosomes, each derived from a different chromosome. OBM Genetics (2017). DOI:10.21926/obm.genet. 1701002 .

Koren A, Tsai HJ, Tirosh I, Burrack LS, Barkai N, Berman J: Epigenetically inherited centromere and neocentromere DNA replicates earliest in S-phase. PLoS Genet 6:e1001068 (2010).

Liehr T, Claussen U, Starke H: Small supernumerary marker chromosomes (sSMC) in humans. Cytogenet Genome Res 107:55-67 (2004).

Locke DP, Hillier LW, Warren WC, Worley KC, Nazareth LV, et al: Comparative and demographic analysis of orang-utan genomes. $\mathrm{Na}$ ture 469:529-533 (2011)

Lomiento M, Jiang Z, D’Addabbo P, Eichler EE, Rocchi M: Evolutionary-new centromeres preferentially emerge within gene deserts. Genome Biol 9:R173 (2008).

Marshall OJ, Chueh AC, Wong LH, Choo KH: Neocentromeres: new insights into centromere structure, disease development, and karyotype evolution. Am J Hum Genet 82: 261-282 (2008).
Murmann AE, Conrad DF, Mashek H, Curtis CA, Nicolae RI, et al: Inverted duplications on acentric markers: mechanism of formation. Hum Mol Genet 18:2241-2256 (2009).

Rocchi M, Archidiacono N, Schempp W, Capozzi $\mathrm{O}$, Stanyon R: Centromere repositioning in mammals. Heredity (Edinb) 108:59-67 (2012).

Seuánez H, Fletcher J, Evans HJ, Martin DE: A chromosome rearrangement in orangutan studied with Q-, C-, and G-banding techniques. Cytogenet Cell Genet 17:26-34 (1976).

Sharp AJ, Cheng Z, Eichler EE: Structural variation of the human genome. Annu Rev Genomics Hum Genet 7:407-442 (2006).

Tolomeo D, Capozzi O, Stanyon RR, Archidiacono N, D'Addabbo P, et al: Epigenetic origin of evolutionary novel centromeres. Sci Rep 7: 41980 (2017)

Turleau C, de Grouchy J, Chavin-Colin C: Pericentric inversion of no. 3, homozygous and heterozygous, and centromeric transposition of no. 12 in a family of orangutans. Implications for evolution (in French). Ann Genet 18: 227-233 (1975).

Ventura M, Antonacci F, Cardone MF, Stanyon R, D'Addabbo P, et al: Evolutionary formation of new centromeres in macaque. Science 316:243-246 (2007).

Wandall A, Tranebjaerg L, Tommerup N: A neocentromere on human chromosome 3 without detectable alpha-satellite DNA forms morphologically normal kinetochores. Chromosoma 107:359-365 (1998).

Wong NC, Wong LH, Quach JM, Canham P, Craig JM, et al: Permissive transcriptional activity at the centromere through pockets of DNA hypomethylation. PLoS Genet 2:e17 (2006). 